\title{
Enfin, je vais à l'école! \\ Une école primaire mixte, dans le Québec des années 1940
}

À ma mère

Micheline Dumont

\section{RÉSUMÉ}

Récit du parcours scolaire d'une fillette au Québec, durant les années 1940, dans une école primaire mixte du Québec, comme l'étaient la majorité des écoles de villages et de petites villes. Alors que la Seconde Guerre mondiale rythme au loin la vie du monde, ce récit nous informe sur l'enseignement avant la grande réforme pédagogiques de 1948, sur les institutrices, sur l'encadrement religieux et sur le rôle positif que joue l'école à cette époque qui précède la télévision, dans une famille où l'instruction est très valorisée.

\section{ABSTRACT}

This is the story of the school path of a little girl of Quebec during the 1940s. Hers was a mixed primary school, as were the majority of schools found in little towns and villages in Quebec. While the Second World War gave far away rhythm to the life of the world, this story informs us about the education offered before the great educational reform of 1948; on the schoolmistresses, the religious frame, and the positive role played by the school in the pre-television period, in a family where education was greatly valued.

Septembre 1941. C'est la guerre, j'ai six ans et enfin, je peux aller à l'école. J'habite Dorion, petite ville de 1000 habitants à l'ouest de Montréal. Terminé le temps où je regarde avec envie les écoliers et les écolières passer devant la maison avec leur sac d'école. Je connais bien l'école : elle est située à l'autre bout de notre petite ville et je passe à proximité chaque dimanche, en faisant la visite rituelle à mes grands-parents. Au surplus, dans l'album de photos familial, il y a une photo datant de 1915 , où tous les écoliers avaient été photographiés devant cette même école avec leurs deux institutrices. Les directives du photographe avaient placé côte à côte la petite Lucile $(5$ ans) et le petit Robert ( 6 ans), mes parents (voir photo 1$)$ : cela nous a toujours semblé, à nous leurs enfants, une irrésistible prédestination! 


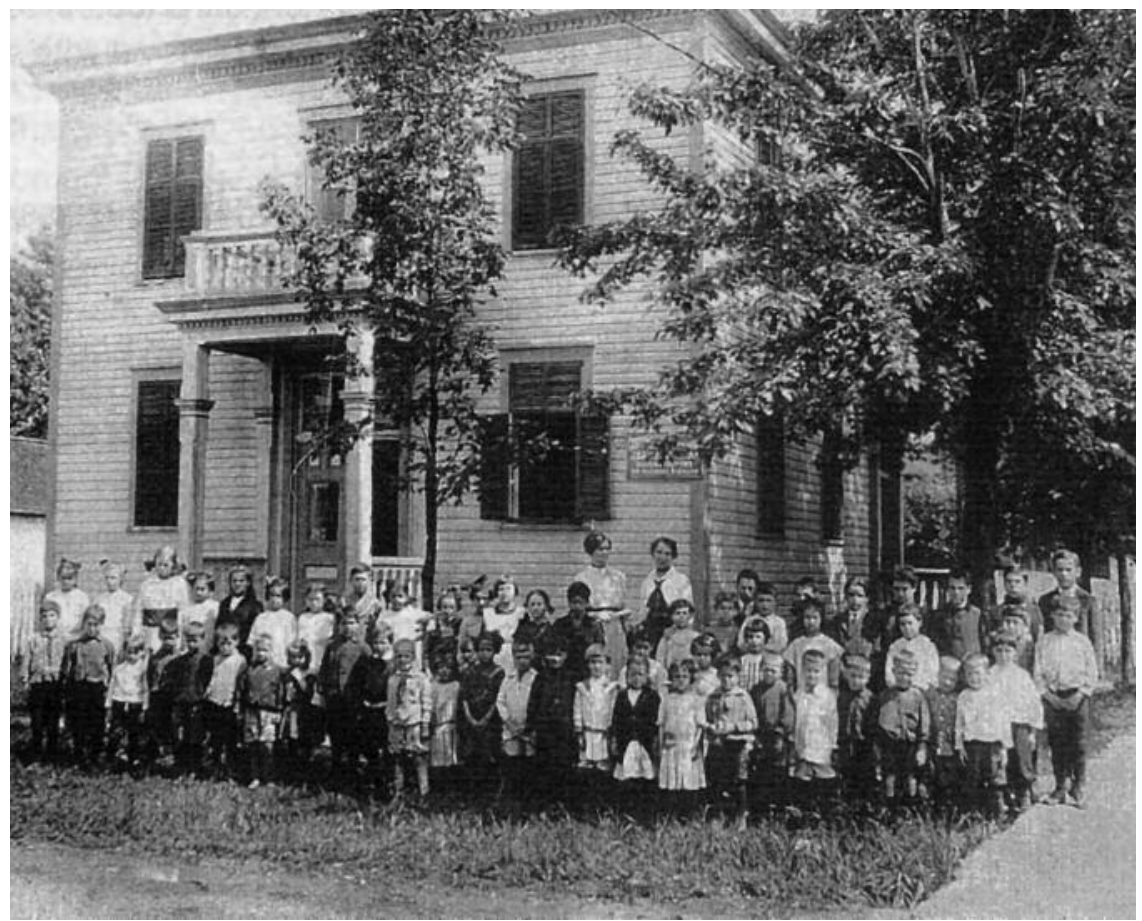

Photo 1 : L'École de Dorion en 1915. Les parents de Micheline Dumont sont les $11^{e}$ et $12^{e}$ élèves de la $1^{\text {re }}$ rangée à partir de la gauche.

C'est une école mixte, même si les enfants sont séparés par sexes dans la cour de récréation. C'est la même école que celle de mes parents, mais elle a été agrandie pour accueillir les enfants plus nombreux et ajouter le cours complémentaire. Des religieuses, trois Sœurs de Sainte-Anne, sont en place depuis 1920, aidées par trois institutrices laïques. Un instituteur a la responsabilité de tous les garçons au-delà de la sixième année, dans une maison voisine. La directrice est une religieuse.

Garçons et filles sont réunis dans quatre classes : première, deuxième, troisième année, classe jumelée de quatrième et cinquième années. Les filles plus grandes se retrouvent dans deux autres classes conjointes : sixième et septième années et une classe complémentaire pour les adolescentes de la huitième à la onzième année. Habituellement, il n'y a qu'une ou deux persévérantes dans cette ultime classe de onzième année. Celle qui y est, en 1941, me semble tellement grande! Jamais je ne pourrai me rendre jusque-là! Les grands garçons sont dans une maison voisine avec monsieur Blais, qui enseigne à tous les garçons de la sixième à la neuvième année. Son autorité et son savoir sont impressionnants. Il est « le maître! ». Dans cette classe, la persévérance scolaire est encore plus faible que chez les filles, car tous les garçons susceptibles d'entreprendre des études classiques sont déjà partis dans les collèges classiques des environs, à Rigaud, à Valleyfield, ou à Montréal. Fréquenter l'école, en 1941, n’est pas obligatoire au Québec. 
Mais, dans ma famille, aller à l'école est une occupation importante, précieuse, incontournable. Dès la première année, je savais que je fréquenterais l'école au moins onze ans. Mes seize oncles et tantes ont tous poursuivi l'école jusqu'à la onzième année. Mon père est ingénieur civil, diplômé de l'École polytechnique en 1932. Ma mère a reçu son "Diplôme d'école modèle ", au pensionnat voisin en 1925. Je ne le sais pas, mais je suis privilégiée. Depuis deux ans déjà, j’étudie la diction!

Titulaire de la première année, Lucille Daoust est adorée des enfants qui l'appellent « Mademoiselle Da-oust-oust » sur ces notes : do, do, do, do, la, fa. Cette institutrice a par la suite révélé que, durant les premières semaines, elle s'occupait surtout à bien dresser son troupeau : le silence dans les rangs, la tenue posturale, le dos bien droit, la piété durant les prières, la propreté des mains et des ongles, les rangs bien alignés pour circuler dans les corridors, les salutations à la maîtresse avant et après la classe : "Bonjour mademoiselle! », le matin, et " Au revoir mademoiselle et merci! », à quatre heures. Sans oublier la cloche de la récréation. Au premier coup, on doit s'arrêter pile, au milieu de notre jeu, quel qu'il soit. Au second, on doit courir en silence s'aligner avec notre classe pour pouvoir entrer en ordre dans l'école. Une fois cette routine bien installée, elle peut enseigner les lettres, les chiffres, le catéchisme, l'écriture, la lecture facilement : ses élèves réussissent à l'exception de quelques cancres irrécupérables. Plus de trente enfants se retrouvent dans sa classe.

Nous avons des pupitres fixés au sol et chaque pupitre reçoit deux enfants. Nous glissons nos livres sous le pupitre dûment percé d'un trou pour recevoir la bouteille d'encre. Mais, en première année, nous n'avons droit qu'au crayon de mine de plomb. Écrire à l'encre représente un art qui ne nous sera permis qu'en troisième année. Je n'oublierai jamais ma compagne de pupitre, dont les minuscules crayons tenaient dans une petite boîte de pastilles, alors que moi j'avais un beau coffre en bois, à deux étages, avec de beaux crayons aiguisés soigneusement par mon père. Nos cahiers d'écriture ont des lignes doubles, pour nous apprendre la régularité dans l'écriture. Ma mère recouvre mes livres avec une légère cuirette rouge vin, cousue sur chaque livre. On lui a accordé une permission spéciale pour le faire car le papier brun est réglementaire. Mon livre de lecture est le même qui a servi à mes tantes et à mes oncles. Il date sans doute de 1925 .

Quatre fois par jour, je fais le trajet qui sépare ma maison de l'école, environ un quart de mille (400 mètres). Je ne mets pas de temps à découvrir des amies, avec qui il est possible de marcher vers l'école en nous tenant par la main et en jacassant à perdre haleine! Durant l'automne, nous cherchons les belles feuilles d'érable rouge que nous pressons dans nos livres. L'hiver venu, nous marchons au-dessus des bancs de neige accumulés de chaque côté de la rue, en faisant attention aux chevaux qui pourraient prendre l'épouvante, apeurés par les rares voitures. Au printemps, nous suivons les rigoles qui ruissellent sur la chaussée détrempée.

Pour atteindre l'école, nous devons traverser les deux passages à niveau où passent quotidiennement des centaines de train. En effet, le CNR et le CPR traversent notre ville à quelque cent pieds l'un de l'autre, sur le trajet Montréal-Toronto et MontréalOttawa. Mon père m’a expliqué que ce sont les lignes les plus importantes du Canada. Le père d'un garçon de notre classe est garde-barrière dans une petite guérite au sommet 
d'une tour. Chaque midi, Gilles monte à la guérite remettre à son père le repas du midi. Nous envions secrètement son immense privilège : il peut actionner la manivelle qui fait descendre les barrières. En 1966, un terrible accident d'autobus scolaire, faisant 20 morts à l'un de ces passages à niveau, incitera les compagnies de chemin de fer à aménager des tunnels sécuritaires.

Un certain matin, au moment où les enfants commencent à circuler, ils sont bloqués par un train de marchandise interminable qui avance lentement. Des centaines de wagons! Sur les plates-formes, des camions, des jeeps, des chars d'assaut, des canons. Les garçons sont surexcités. Bientôt, la grande majorité des enfants sont massés près de la clôture, ainsi que les institutrices sans oublier les religieuses dans leur taxi qui les amène du village voisin où elles habitent. Le train se dirige vers Montréal et, sans doute après, vers Halifax, pour livrer cette précieuse cargaison aux Alliés. Ce matin-là, la prière pour que "Dieu accorde la paix aux Alliés " prend une signification particulière. Un autre jour, les enfants se pressent aux fenêtres de l'école pour voir défiler un régiment de "Black Watch », qui nous font rigoler avec leurs jupettes. La maîtresse nous explique que la jupe qu'ils portent se nomme un " kilt » et qu'avant de s'engager sur le pont ils vont briser le pas, afin de protéger la structure du pont, car des centaines de soldat marchant au pas peuvent causer sa chute. Notre maîtresse sait tout : elle peut tout expliquer!

Chaque midi, tous les enfants s'installent à genoux, à l'envers sur les bancs, pour regarder le crucifix à l'arrière de la classe. On récite le chapelet. Pendant la prière, la maîtresse circule entre les rangs pour réparer les chapelets brisés. Le mien l'est toujours : il me manque des "Je vous salue marie » et un "Gloire soit au père ». Le curé vient aussi nous faire des leçons de catéchisme. Quelque temps avant la première communion, on procède à un exercice pour vérifier si on a bien appris les formules de la confession. À tour de rôle, on défile devant lui, dans un endroit interdit, la cuisine des religieuses, en prenant bien soin de ne pas dire nos vrais péchés. Je me suis accusée d'avoir tué une mouche et d'avoir regardé au plafond! Le jour de la première communion, j'ai une très belle robe cousue par ma mère, qui est une véritable fée, et un voile blanc. Je ne me souviens d'aucune émotion particulière. C'est un rituel, sans plus. Je reçois en cadeau un petit missel et des images pieuses que je distribue solennellement à toute la parenté. Je fais également ma confirmation. L'évêque vient tous les quatre ans et sa tournée correspond à mon passage en première année. Je fais donc ma confirmation en même temps que mes deux cousins plus âgés, et cela me remplit de fierté. Et je garde un souvenir brûlant du soufflet retentissant que m’a asséné l'évêque.

L'institutrice de la deuxième année est une institutrice chevronnée, une amie de mes tantes. Elle a la réputation d'être très sévère. Plus tard, lors de mes recherches en histoire de l'éducation, je découvrirai des articles qu'elle avait publiés dans La petite école, le premier journal des institutrices rurales. Son nom est Emma-Maria Lalonde. Mais les garçons la surnomment «Emma-Barouette », pour quelque raison mystérieuse. Je ne la trouve pas sévère car je suis très appliquée : je fais soigneusement mes devoirs et je sais mes leçons : ma mère y voit tous les soirs. La maîtresse suscite une grande émulation entre les garçons et les filles. Nous sommes cinq, deux garçons et trois filles, qui nous disputons la première place. 
Mais en novembre 1942, catastrophe! L'école passe au feu. De ma chambre, j'entends mon père qui est allé au feu, et raconte l'incendie, les pompiers. Qu'allons-nous devenir? Les commissaires trouvent des locaux de fortune. Après quelques jours de vacances forcées, ma classe se retrouve dans la salle municipale. Une autre classe est installée sur la scène, derrière les rideaux. Une autre dans la salle du conseil municipal, dans une pièce au-dessus. Le chauffage est difficile et la maîtresse nous réunit autour de la fournaise pour la répétition des leçons. C'est vraiment la cacophonie dans la salle municipale. Finalement, au grand désespoir des parents et à la grande satisfaction des enfants, on décide d'envoyer les enfants à l'école par demi-journées. L'école sera reconstruite pour la somme de 20490 \$, mais ne sera disponible qu'en septembre 1944. Ma petite sœur qui commence l'école en septembre 1943 doit aller à l'école dans un garage!

La troisième année se passe donc dans la salle municipale. Je conserve la même institutrice qui monte de classe avec moi. J'avais pourtant hâte d'avoir une nouvelle maîtresse, mais, comme je la connais, j’apprends mieux. En troisième année, on commence la rédaction. Pour la première fois de ma vie, je dois écrire un texte qui vient de ma tête. Cette perspective me semble très attrayante. Comme de juste, on nous propose comme thème "la rentrée des classes ". Je n'ai jamais oublié la dernière phrase de ma première rédaction : "Le soleil brille et tout le monde est heureux! » Au verso de tous mes cahiers se trouvent les fameuses tables de multiplication que je ne maîtrise jamais complètement. Avec mes doigts, qui pianotent avec énergie en cachette, je réussis toutefois à trouver les bonnes réponses.

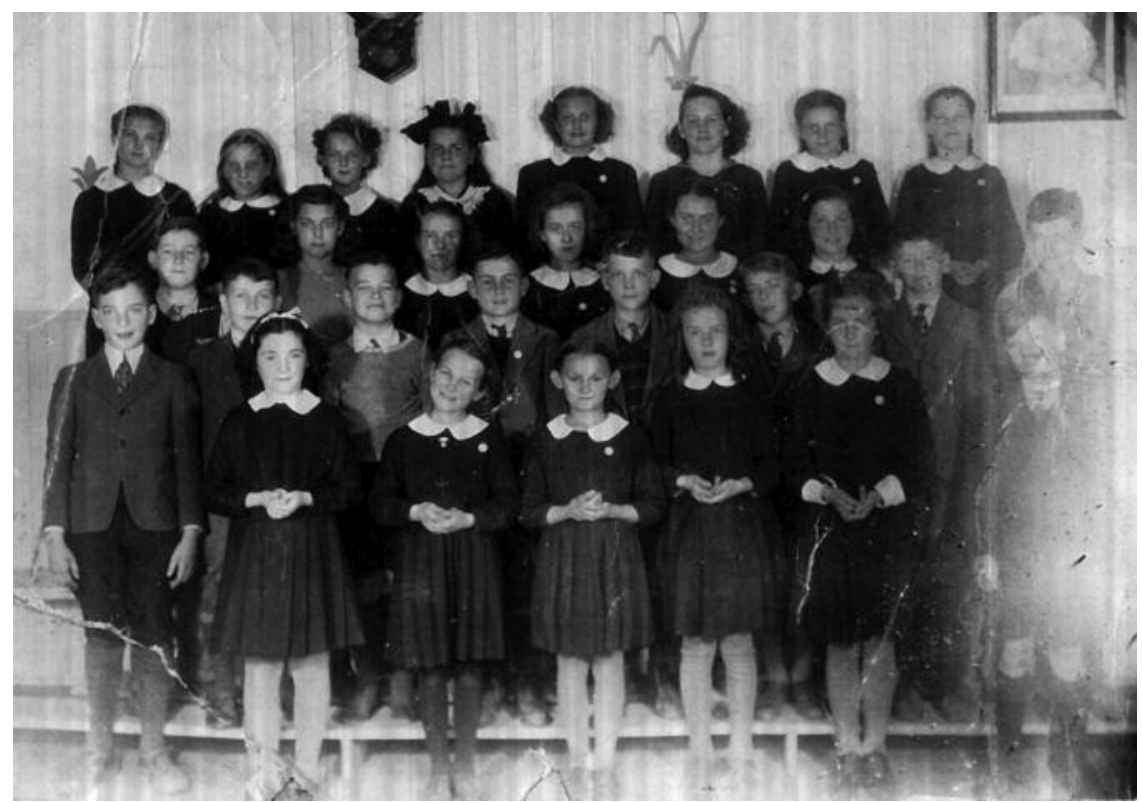

Photo 2 : Classe de $4^{e}$ et de $5^{e}$ années de l'École de Dorion. 
Grande première : je dois écrire à l'encre. Depuis longtemps déjà, j’observe mon père qui écrit à l'encre dans ses livres de compte, dans ses albums de timbres, qui appose sur mon bulletin sa signature illisible (À ma grande honte, j'estime que la maîtresse devrait lui donner des leçons d'écriture!). Surtout, il utilise le buvard qui me semble une opération magique : il imprime l'écriture à l'envers! Expérimentée, la maitresse n'admet à cette initiation que quelques enfants à la fois. Comme mon écriture n'est pas très soignée, je ne passe pas la première. Mais mon tour arrive enfin. Je m’installe, avec le précieux buvard à proximité. J'écris mes premiers mots avec le porte-plume et je frotte consciencieusement ma page avec le buvard. Désastre : mes mots au lieu de s'imprimer sur le buvard, se sont répandus à travers la page. Je mets quelque temps à maîtriser l'art du buvard, beaucoup plus difficile pour moi que l'art d'écrire à l'encre. Mais surtout, en troisième année, je peux commencer à lire avec facilité. Je fréquente la bibliothèque paroissiale qui possède la collection complète des romans de Maxine, les romans du Père Hublet et les romans scouts de la collection "Signe de piste ». Je me rends chez ma grand-mère lire les volumes de l'Encyclopédie de la jeunesse. La section "Récits, contes et légendes " m’ouvre la porte de l'univers merveilleux de la fiction. Quand je lis, je disparais dans mon fauteuil : le monde cesse d'exister autour de moi. Voyant cet intérêt, ma mère choisit les romans de la Comtesse de Ségur pour Noël et mes anniversaires. Bientôt, je les posséderai tous, les vingt-six. Mon préféré est Un bon petit diable. Ma mère m'achètera aussi, avec le temps, les romans historiques de MarieClaire Daveluy: Les aventures de Perrine et Charlot, en six volumes, et les romans de T. Trilby, mes préférés, les albums de La Semaine de Suzette où on retrouve les romans de Berthe Bernage, Les aventures de Sir Jerry, et la bande dessinée de Bécassine. À la même période, ma mère a pris la décision qu'à la place de la diction, j'étudierais le piano.

À l'école enfin reconstruite et agrandie, je passe en quatrième année, avec une religieuse, Soeur Marie Angéline, un ange certainement. Nous l'adorons. Les élèves de cinquième année sont avec nous. Nous sommes dix-huit filles et treize garçons. Pour la première fois, nous avons des pupitres individuels dont le couvercle se soulève. Cette année, la liste de mes manuels me semble interminable : catéchisme, grammaire, lecture, histoire, connaissances usuelles, géographie, économie domestique, mathématiques, toisé (formules des formes géométriques). La cuirette rouge vin de ma mère est toujours au rendez-vous pour en prendre soin. Je découvrirai plus tard que chaque manuel est écrit par une congrégation religieuse différente : les Clercs de Saint-Viateur, l'histoire du Canada; les Frères Maristes, la géographie; les Sœurs de la Congrégation, la lecture; les Sœurs de Sainte-Anne, les connaissances usuelles. Pour la première fois, mes cahiers d'écriture n'ont que des lignes simples mais je dois utiliser le transparent, qui assure l'inclinaison correcte des lettres. Commence alors mon combat personnel contre les transparents et l'écriture uniforme. Je n'y arriverai vraiment jamais. Grande nouveauté : je dois porter un uniforme, une robe noire à col blanc, que ma mère doit nettoyer chaque samedi en utilisant du thé. Les bas longs sont obligatoires et nous envions les Anglaises qui vont à l'école en bas courts. (photo 2)

Ma classe est à l'étage. Elle est décorée des tableaux historiques de Desrosiers et Bertrand, 26 aquarelles de Lagacé qui racontent l'Histoire du Canada, avec l'accent 
sur tous les épisodes militaires et religieux de la Nouvelle-France. J'ai tellement rêvé en regardant ces tableaux : de là origine sans doute mon goût de l'histoire. De mon pupitre, je peux apercevoir la rivière des Outaouais qui coule à proximité. C'est la source de mes fréquents passages dans la lune. J'aime toutes les matières et il m'arrive souvent d'aller fureter dans les pages qu'on n'a pas encore étudiées. Je suis fascinée par les leçons d'anatomie et j'ambitionne d'apprendre par cœur le nom des 208 os du corps humain. De même pour les comtés de la province de Québec : mais j’ai plus de difficulté. Les noms de comtés anglais me donnent beaucoup de mal. Certains mots me font rêver : Chicoutimi, Maskinongé, Témiscouata. Personne ne m’explique que ces mots ont une origine amérindienne. Il y avait 14000000 d'habitants au Canada. Encore aujourd'hui, c'est le chiffre qui me vient à l'esprit quand on évoque la population canadienne. Sont également gravés pour toujours dans ma mémoire : le gouverneur-général, Athlone; le premier ministre à Ottawa, Mackenzie King; le premier ministre à Québec, Duplessis, tout juste élu avant la rentrée scolaire.

Mais on ne doit pas oublier que c'est toujours la guerre. Parfois, les enfants arrivent à l'école avec des histoires terribles : la "Emmepie », ( il s'agit de la Military Police, mais j'ignore à ce moment-là le sens de cette expression menaçante), est venue chez les X. Pour éviter l'enrôlement, l'un s'est caché entre les cordes de bois du boulanger Gaucher, juste au coin de la rue. Un autre s'est caché dans les piles de pneus du garage Dumoulin. Un autre s'est caché dans l'Ile-aux-pins. Ses petits frères doivent lui porter de la nourriture en chaloupe. La guerre, pourtant si lointaine, reste présente pour les enfants : 87 jeunes gens de Dorion ont participé à la Seconde Guerre mondiale. Chacun et chacune a son frère, son oncle, son cousin à la guerre. Un frère de mon père part pour l'Angleterre en mars 1945, au grand désespoir de ma grand-mère.

L'entrepreneur qui a reconstruit l'école a dressé dans la cour un mât. La directrice en profite pour instaurer un nouveau rituel : le salut au drapeau. Tous les vendredis, l'école entière se réunit et chante $\hat{O}$ Canada. Puis, le plus grand garçon de l'école prononce à voix forte : «À mon drapeau, je jure d'être fidèle! » Tous les élèves en frissonnent!

Cette année-là, la Jeunesse étudiante catholique fait son entrée à l'école. Nous participons à la semaine étudiante par un défilé dans les rues de la ville. La responsable crie dans son porte-voix : «Étudiants! ». Nous répondons : «Cent pour cent! ». Nous chantons un hymne martial : Joie Difficile! , écrit sur un air de Beethoven, la Coda du quatrième mouvement de l'Héroïque, (je l'ai découvert longtemps après). Par quelle grâce 200 enfants qui ignoraient même l'existence de Beethoven pouvaient-ils chanter cet air avec justesse et détermination? Qui étaient les audacieux responsables qui avaient eu cette initiative? Pour cet événement, les classes et les corridors sont décorés par les centaines de tulipes multicolores que nous avons fabriquées.

Les religieuses, qui ne veulent pas être en reste, nous embrigadent également dans la "Croisade eucharistique ». Cette association nous semble plus intéressante parce qu'il y a un uniforme : une cape et un béret de satin blanc décorés d'une croix rouge. Garçons et filles les portent durant les cérémonies religieuses à l'église paroissiale. Et l'école entière s'y retrouve si souvent : le mois du rosaire en octobre, le chemin de la croix chaque vendredi, le mois de Saint-Joseph en mars, le mois de Marie en mai, le premier 
vendredi du mois, les rogations, les jours Saints. Quand toute l'école chante à voix forte sur un air militaire :

En avant marchons! En avant marchons!

Soldat du Christ à l'avant-garde!

En avant marchons, en avant marchons!

Le seigneur nous regarde.

En avant bataillon!

C'est vraiment l'Église triomphante! Je suis impressionnée à chaque fois que le chant retentit dans la petite chapelle qui sert d'église paroissiale. Le samedi, les filles retournent à l'église pour observer les mariages : nous admirons les robes, les fleurs. Nous remplissons le jubé de nos bavardages.

Le 7 mai 1945, la cloche sonne pour appeler tous les enfants dans la cour. On nous place en rangs, devant chaque titulaire de classe. Que se passe-t-il? La directrice de l'école nous annonce solennellement que la guerre est terminée. On hisse le drapeau, le red ensign, et on chante l'hymne national Ô Canada! Et on nous donne congé. Vive le Canada! Vive l'Angleterre! Vive la France!

Les grandes vacances sont consacrées aux jeux de toutes sortes et au travail domestique. Ma mère, qui nous libère de toutes les tâches ménagères durant l'année scolaire, " parce que l'école, c'est plus important que le ménage ", distribue les tâches à ses filles. Toutes doivent contribuer, même la plus petite : elle doit balayer le perron d'en arrière. Vaisselle, lavage, repassage, ménage, cuisine, nous apprenons nos futures responsabilités. Je commence aussi à garder mes petites sœurs pour vingt-cinq sous la soirée. Mais je ne vois pas cet argent : il est inscrit dans un carnet par mon père. Quand la somme requise sera atteinte, je pourrai obtenir un magnifique vélo CCM, modèle 1945. Mais nous allons également nous baigner dans la rivière des Outaouais, dans un bain flottant que mon grand-père a fait aménager au large. Et nous montons des pièces de théâtre. Ma mère a aménagé une boîte de costumes, et au gré de notre fantaisie, nous devenons tour à tour metteures en scène, réquisitionnant quelques actrices, pour un scénario et des dialogues improvisés sur place. Les spectatrices regardent de bonne grâce, sachant qu'elles seront actrices ou metteure en scène le coup suivant. Combien de "pièces " avons-nous ainsi jouées? Certainement des centaines!

À l'été de 1948, les garçons nous informent de la tenue des jeux Olympiques à Londres. Ils décident d'organiser une compétition, chaque enfant représentant un pays. Je représente les États-Unis. Nous devons participer à toutes les épreuves d'athlétisme : sauts, courses. Il a fallu une grande journée pour nous organiser, faire les drapeaux (merveilleux dictionnaire Larousse qui a fourni les modèles), préparer les feuilles de pointage, préparer des bancs pour les rares spectateurs. M'étant foulé la cheville au saut en longueur, j'ai donné à mon pays le dernier rang, même si j'ai tenu à boitiller à toutes les courses.

La cinquième année ressemble à s'y méprendre à la quatrième : même classe mais une titulaire différente, Sœur Marie Anne-Fébroni. Les occasions de tomber dans la 
lune se multiplient, mais je deviens versée malgré tout dans l'analyse grammaticale et dans l'analyse logique. J'adore étudier l'histoire et la géographie. L'école est devenue une routine qui m'intéresse mais offre très peu de surprises. Notre institutrice nous incite à aller à la messe tous les jours durant le carême. Quand je pars pour l'église, il fait encore noir. J'ai un peu peur : j'aperçois des chiens, des voleurs, des loups. Mais au retour de la messe, je vois bien que ce sont des poubelles, des buissons, des souches d'arbre. Comment se fait-il que, le lendemain, je continue de craindre les voleurs et les chiens?

La seule nouveauté est suscitée par la chorale. Les filles se rassemblent après l'école sous la direction d'une musicienne et préparent des messes à quatre voix, des motets, et même, pour la fête de Pâques, un oratorio. Grâce à nous, les saluts du Saint-Sacrement sont devenus de belles cérémonies. Et quel plaisir d'assister aux offices dans le jubé plutôt que dans les bancs!

Chaque année, nous avons la visite de l'infirmière, qui répète inlassablement les mêmes conseils : se brosser les dents, se nettoyer les ongles, se laver matin et soir, ne jamais mettre un tricot sous notre uniforme, porter des jarretières qui ne serrent pas trop la cuisse, prendre de l'huile de foie de morue durant l'hiver, vérifier si nous avons des poux. Elle vérifie notre vision et, cette année-là, en 1946, elle recommande à mes parents une visite chez l'oculiste. Je dois porter des lunettes et ces lunettes ressemblent à celles du pape Pie XII.

En mai, notre famille est surexcitée par le retour de mon oncle soldat. Certes, il n'a pas connu la « vraie guerre " puisqu'il est arrivé en Angleterre quelques semaines avant la fin des combats en Europe. Mais il a fait l'armée d'occupation en Allemagne. Nous allons l'accueillir à la gare en criant « Welcome! Welcome! ». Plusieurs familles vivent la même excitation.

Puisque maintenant nous sommes dans les classes des religieuses, la fin des classes en juin nous incite à chanter, avec les garnements de l'école :

Vive les vacances,

Au diable les pénitences!

Mettons l'école en feu

Pis les sœurs dans le milieu!

Je monte enfin en sixième année. Grande nouveauté à l'école : la congrégation des Clercs de Saint-Viateur vient prendre en charge tous les garçons à partir de la quatrième année. Mon père chicane un peu : il n'aime pas les frères mais, comme il n'a que des filles, il ne peut rien dire! Seules les trois premières classes resteront mixtes. De toutes manières, je devais monter dans une classe de filles. Quelle joie : nous retrouvons Sœur Angéline! La belle année que nous allons passer! Elle se déroule en effet sans histoire, ou presque. Deux aventures émergent parmi mes souvenirs : on me choisit pour interpréter un rôle dans une pièce de théâtre. Je dois jouer une aveugle. Les "grandes " ont les autres rôles. On me conseille de fermer les yeux pour bien montrer que je suis aveugle. Durant les répétitions, je m'entête à ne pas le faire parce qu'une de mes cousines est aveugle et, elle, elle ne ferme jamais les yeux. Mais finalement, le grand jour 
arrive et j'accepte de me fermer les yeux durant la représentation. Une autre fois, on nous emmène visiter un orphelinat à Montréal. Nous assistons au déjeuner des garçons de 2 ans. Quel spectacle! Deux religieuses habillent et font manger une quarantaine d'enfants. Parmi eux, un petit noir. D'où peut-il bien venir?

Ma mère répond avec embarras aux questions que je lui pose. Par contre, elle prend une initiative intéressante. Tous les samedis, avec ma sœur plus jeune et nos deux cousins, elle nous fait chanter dans les Albums de la Bonne chanson de l'abbé Gadbois, qu'on trouve alors dans presque toutes les familles. Elle se met au piano, et nous enseigne plusieurs chansons par semaine. C'est une activité que nous adorons et que nous faisons avec enthousiasme.

En 1947 enfin, j'atteints le septième année, année très importante, celle du Certificat d'études. Hélas! Sœur Angéline retourne en 4 et 5, et je me retrouve avec Sœur Julienne-de-Provence, nouvelle titulaire de 6 et 7 . Pour mon malheur, elle me prend en grippe. Elle corrige sévèrement mes rédactions qu'elle trouve trop fantaisistes. Je m'entends aussi de moins en moins avec mes compagnes qui sont plus vieilles de caractère que moi. Elles s'intéressent aux garçons, qui sont le cadet de mes soucis. J'aime les garçons quand ils jouent avec nous durant les vacances. Autrement ... Je me suis trouvé un autre trajet pour aller à l'école pour mieux rêvasser au long du chemin. Il m'arrive d'aller rêver seule au bord des rapides de la rivière des Outaouais. Cette année-là, c'est vraiment la routine : j'ai l'impression de ne plus rien apprendre. J'ai beau fureter dans mes manuels, il n'y a plus grand chose d'intéressant.

La visite de l'infirmière cause un petit drame. Comme ma mère a fait couper mes cheveux, j'ai une coiffure à la mode, un "brush cut ", qui déplait à la religieuse parce que j'ai une mèche qui tombe dans la figure. Elle m'oblige à mettre une "bobby pin " pour la relever. Quand l'infirmière entre dans la classe, ostensiblement, en regardant la religieuse bien en face, j'enlève la «bobby pin » et je la lance en arrière de la classe. Ce geste anodin de révolte me mérite une sévère punition. Mais ma mère réussit à me consoler.

Je dois me présenter aux examens diocésains de catéchisme. Il a fallu mémoriser toutes les réponses du Petit catéchisme. J'obtiens la note de $100 \%$ et mon premier diplôme! Sœur Julienne de Florence peut bien me disputer : elle ne pourra pas dire que je ne sais pas mon catéchisme!

Certes je réussis bien à l'école, mais mon univers mental est limité. Je suis allée à Montréal en train régulièrement : c'est ma seule destination. Ma mère m'a montré les voies ferrées qui mènent à Québec, celles qui mènent à New York. Mais ces villes demeurent pour moi des villes mythiques. Je connais bien sûr la direction de Toronto et d'Ottawa : mais je n'y suis jamais allée. Mon seul regard sur ailleurs me vient d'incursions régulières sur les illustrations du National Geographic Magazine auquel mon père est abonné. Incursions coupables d'ailleurs, à cause de tous ces indigènes tout nus! Je ne vais jamais au cinéma : il est interdit aux enfants de moins de 16 ans. Mais je me souviens d'un film présenté à la salle municipale, Le Carrefour des enfants perdus ( avec Serge Reggiani, alors adolescent), film qui me bouleverse. Je reviens chez moi en sanglotant. Je me souviens aussi d'une pièce de théâtre. Les Compagnons de Saint-Laurent, 
la célèbre troupe du Père Émile Legault, vivent dans la région. Ils viennent un jour présenter Le jeu de l'amour et du hasard de Marivaux, qui est pour moi un enchantement, une porte ouverte sur un univers différent qui reste encore inaccessible.

Quand arrivent enfin les examens du "Certificat ", je suis prête, sauf en économie domestique que nous n'avons pas étudiée du tout. La question fatidique arrive : "Quelles sont les différentes étapes pour faire du neuf avec du vieux? » Je ne l'ai pas appris dans le livre, pour la simple raison que nous n'avons jamais ouvert ce livre. Mais j’ai vu ma mère à l'œuvre tout au long de la guerre. Elle est une spécialiste du recyclage en couture. Alors, je décris les gestes de ma mère : découdre, laver, repasser, fixer le nouveau patron avec des épingles, découper, recoudre, et j'obtiens la meilleure note! Ça y est : j'ai obtenu mon Certificat de septième année. À la distribution des prix, je reçois un bel album Histoire de France racontée aux enfants, par René Ristelhueber, ministre de France. Ce livre va bien sûr nourrir ma passion pour l'histoire. Des centaines d'enfants ont dû recevoir cet album qui avait été édité à Montréal en 1946 et illustré par un peintre québécois, Maurice Raymond. Mais je pense bien qu'aucun ne l'a lu aussi souvent que moi! Je découvrirai plus tard que cet historien était ministre plénipotentiaire du Gouvernement de Vichy à l'Ambassade de France à Ottawa.

Autre expérience charnière de cette fin d'école primaire, j'accepte de participer, durant les vacances, à un Camp de JEC, pour mieux participer à ce mouvement d'action catholique. J'y découvre un autre visage de la religion, basé non pas sur la piété avec laquelle j'ai de sérieux problèmes mais sur l'engagement, que je découvrirai progressivement au cours des années suivantes. Ce sera le premier d'une série de camps qui vont changer ma vie.

Pour moi l'école primaire est bel et bien terminée. Il était temps! Pour mes petites sœurs qui me suivent, dès l'année suivante, de nouveaux programmes seront adoptés dans toutes les matières, avec toute une série de nouveaux manuels. Je suis passée à travers l'école primaire juste avant cette transformation. Je suis une enfant de l'ancien régime! D'ailleurs, à Dorion, la loi de l'obligation scolaire de 1943 a bouleversé la situation, puisque tous les enfants doivent désormais rester à l'école jusqu'à quinze ans. L'école devient trop petite. Et le baby boom est en pleine action : plus de 312 enfants sont nés entre 1948 et 1953. En 1954, plus de 160 enfants vont à l'école dans des locaux de fortune : dans le vieux presbytère, dans un magasin, dans une salle de réunion. Cette transformation se produit alors que la ville est déchirée par des conflits pour la création d'une nouvelle paroisse, la construction de deux nouvelles églises. En 1960, un incendie détruit la vieille école qui datait de 1893 et avait été agrandie tant de fois. Les Clercs de Saint-Viateur se retirent en 1959; les Sœurs de Sainte-Anne en 1964. La révolution scolaire des années 1960 se charge de modifier pour toujours le paysage scolaire de Dorion.

Avant de terminer ce rappel de mes années d'école primaire, il me reste un hommage à rendre à ma mère. L'école de Dorion était sans doute une école dans la moyenne, ni exceptionnelle, ni désastreuse : j'y ai reçu un bon enseignement. Mais c'est surtout l'encadrement de ma mère qui en a fait pour moi une expérience de découvertes et d'apprentissages féconds. Je lui dois beaucoup. 